\title{
CONTRIBUTION TO THE KNOWLEDGE OF CRYPTOPHAGIDS (COLEOPTERA CRYPTOPHAGIDAE) FROM IRAN
}

\author{
(*) Departamento de Biología Animal, Facultad de Biología, E - 15782 Santiago de Compostela, Spain. E-mail \\ josecarlos.otero@usc.es \\ (**) Department of Plant Protection, Yadegar - e-Imam Khomeini (RAH) Shahre Rey Branch, Islamic Azad University, Tehran, \\ Iran.E-mail: hghahari@yahoo.com \\ (***) Via De Reggio, 142 - 72021 Francavilla Fontana (Brindisi), Italy. E-mail: angelini_fernando@libero.it \\ ( $\left.{ }^{\circ}\right)$ Correspondence: josecarlos.otero@usc.es
}

Otero, J.C., Ghahari, H, Angelini, F. - Contribution to the knowledge of cryptophagids (Coleoptera Cryptophagidae) from Iran.

This paper summary the Cryptophagidae species from Iran and provides new data on the distribution. Seven species are newly recorded for the fauna of Iran: Cryptophagus intermedius Bruce, 1934; Cryptophagus opacosericeus Reitter, 1896; Cryptophagus pallidus Sturm, 1845; Cryptophagus quadrimaculatus Reitter, 1877; Cryptophagus setulosus Sturm, 1845; Atomaria (Atomaria) fasciata Kolenati, 1846 and Atomaria (Atomaria) munda Erichson, 1846.

KEY WorDS: Coleoptera, Cryptophagidae, distribution, new record, Iran

\section{INTRODUCTION}

Cryptophagidae is a Coleoptera family with about 1,000 described species placed in approximately 50 genera and represented in all biogeographic regions. Both adults and larvae of silken fungus beetles are commonly found on mold, fungi, under bark, as well as in decaying vegetation. Some genera are characterized by inquilinism with termites and social hymenopterans (Apidae, Vespidae and Formicidae) (LESCHEN, 1999; Otero et al., 2001; LJUBARSKY \& PERKOVSKY, 2011). Most cryptophagids are nidicolous beetles; they are one of the most abundant beetles in the nests and burrows of rodents, birds and social insects (LJUBARSKY, 1992).

Iran forms a large part of the Iranian plateau, and covers an area of $1,623,779 \mathrm{~km}^{2}$. It is bordered to the north by the Caucasus Mountains, Middle Asian natural regions, and the Caspian Sea (-27 m below sea level); to the west by the Anatolian and Mesopotamian regions; to the east by the eastern part of the Iranian plateau (Afghanistan and adjacent west Pakistan) and the Baluch-Sindian region; and finally to the south by the Persian Gulf and Gulf of Oman, which are connected by the latter to the Indian Ocean (Fig. I). Climatologically, Iran is a predominantly arid and semi-arid country, but the northern slopes of the Alburz ranges and the Caspian lowland receive 800 to $2000 \mathrm{~mm}$ annual rainfall, making them the most humid parts of the country. The Dasht-e Kavir and Dasht-e Lut deserts are the driest areas with an annual precipitation of less than $150 \mathrm{~mm}$. The highlands receive between 250 and $800 \mathrm{~mm}$ (ZEHZAD et al., 2002).

Faunistic knowledge for Iran is limited. JOHNSON (1993) and LJUBARSKY (2000) give some information. Six Cryptophagidae species have also been listed as agricultural pests in the country (MODARRES AwAL, 1997). In addition, several species are listed in the Catalogue of Palaearctic
Coleoptera (JOHNSON et al., 2007) without indicating of locality .

This paper which its aim is cataloging of all the data on Iranian Cryptophagidae, is a continuation of the series of checklists of Coleoptera of Iran (LEGALOV et al., 2010; LASON \& GHAHARI, 2013; BUNALSKI et al., 2014; BARTOlOzZI et al., 2014; GHAHARI et al., 2015; GHAHARI \& NOVAK, 2015).

\section{MATERIAL AND METHODS}

Based on literature and new date sampling sites Cryptophagidae beetles found in Iran (Table 1) were included and summarized by province.

The first author investigated and determined beetle species from different depositories:

- Muséum d'Histoire Naturelle, Géneve, Switzerland: MHNG;

- Hungarian Natural History Museum, Budapest, Hungary: HNHM.

Note: "no locality cited- this is locality for species, which were listed in JOHNSON et al. (2007) without indicating of locality.

\section{RESULTS AND DISCUSSION}

Family Cryptophagidae Kyrby, 1826

Subfamily Cryptophaginae Kirby, 1826

\section{Cryptophagus acutangulus Gyllenhal, 1827}

EXAMINED MATERIAL - East Azarbaijan province, Sofian, 5.VI.1975, 1ex; Bostanabad, 25.VI.1975, 1 ex; Fars province, Dasht-e-Arjan, 1.VI.1974, 1 ex; Northern 


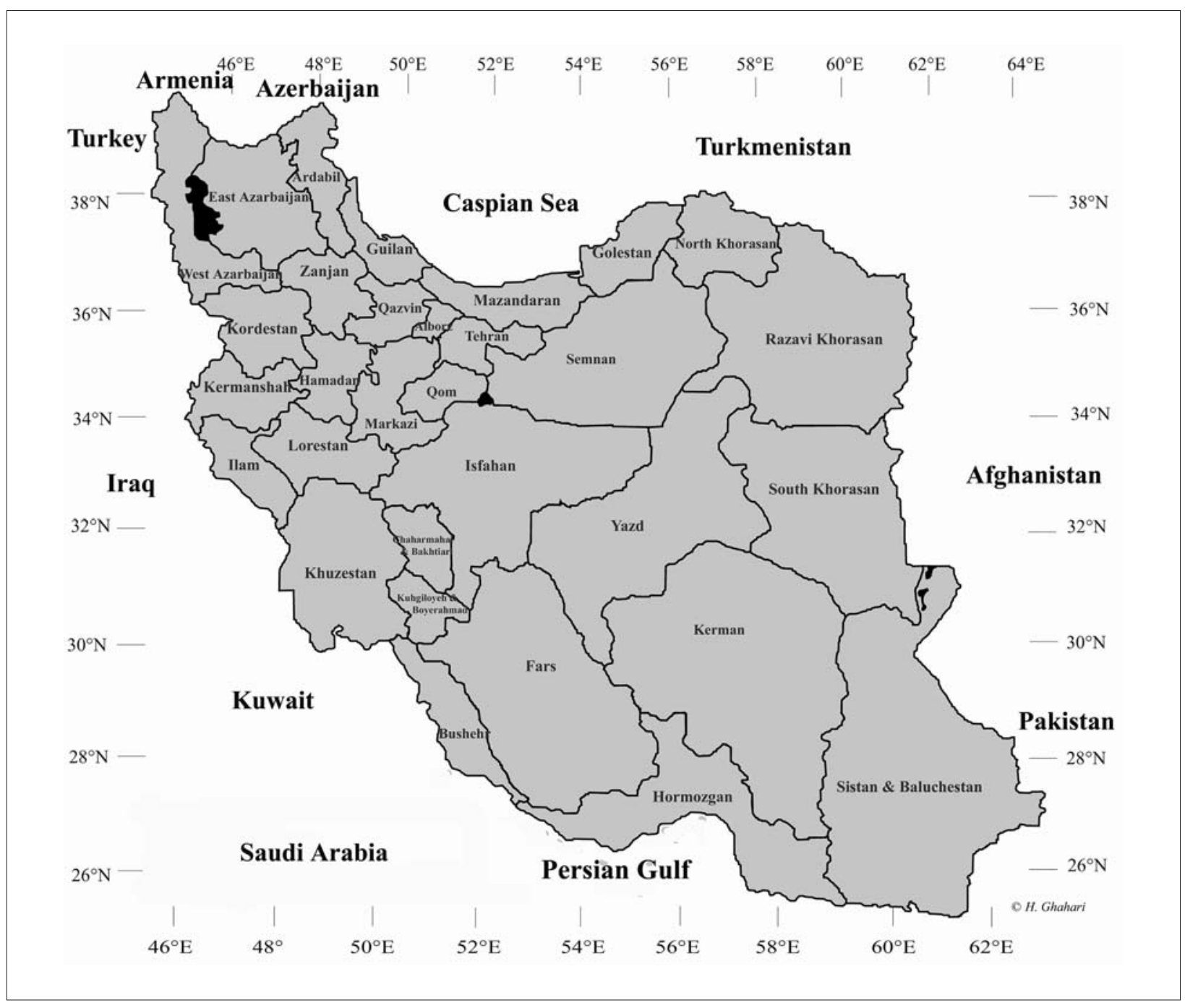

Fig. I - Map of Iran

Khorasan province, Bodjnourd, 26.VII. 1974, 1 ex (leg. A. Senglet, MHNG).

GENERAL DISTRIBUTION - Species present in Holarctic, Neotropical and Oriental Regions (JOHNSON et al., 2007; OTERO, 2011; LJUBARSKY, 2014).

Host - Both larvae and adults feed on fungal spores and hyphae (Psaliota campestres L., Merulius lacrymans (Wolf.), Penicilium glaucum Horr, etc.) (OTERO, 2011).

\section{Cryptophagus araxicola Reitter, 1889}

Distribution IN IRAN - East Azarbaijan (ModarRes AWAL, 1997).

GENERAL DISTRIBUTION - Armenia, Azerbaijan (JoHNSON et al., 2007).

Host - Fungus and on dried fruits (in damp granaries) (ModArRes Awal, 1997).

\section{Cryptophagus bruckii Reitter, 1875}

DISTRIBUTION IN IRAN - Iran (no locality cited) (JOHNSON et al., 2007).

General distribution - Albania, Armenia, Bosnia Herzegovina, Spain, Georgia, South Russia, Afghanistan, Iran, Israel, Jordan, Pakistan, Syria, Turkmenistan, Turkey, Uzbekistan, China (Johnson et al., 2007; Otero, 2013; LJUBARSKY, 2014).

Cryptophagus cellaris (Scopoli, 1763)
Distribution In Iran - East Azarbaijan (ModarRes AwAL, 1997).

NEW DATA - East Azarbaijan province, Sofian, 5.I.1975, 2 ex; Hamadan province, Ganznamh, 2100 m, 4.VII. 1974, 3 ex; Tehran province, Tehran, 2.IX.1973, 1 ex (leg. A. Senglet, MHNG).

General distribution - Cosmopolitan species. Europe, Atlantic Islands, North Africa, Egypt, Asia (Woodroffe \& CoOmBs, 1961; Johnson et al., 2007; OTERO, 2011; 2013).

Host - Fungus and on dried fruits (in damp granaries) (Modarres Awal, 1997).

\section{Cryptophagus cellaroides Reitter, 1921}

Distribution IN IRAN - Tehran, 17.V.1938, 1ex (LJUBARSKY, 2000)

General distribution - South European Territory (Kavkaz), Azerbaijan, Iran, Central Asia, (JoHnson et al., 2007; LJUBARSKY, 2000; OTERO, 2013).

\section{Cryptophagus dentatus (Herbst, 1793)}

EXAMINED MATERIAL - Mazandaran province, Nowshahr, 3.VIII. 1974, 1 ex; Mazandaran province, route de Tchorth, 1300 m, 5.VIII.1974, 1 ex (leg. A. Senglet, MHNG); Kuhgiloyeh \& Boyerahmad province, $3 \mathrm{~km} \mathrm{~N}$. Ssaht, N 53 22’21" E 319', 2700 m, 10.V.1998 (leg. Fabian-Szekely, 
Table 1 - NI: New record for Iran.

\begin{tabular}{|c|c|c|}
\hline Species & Notes & Provinces \\
\hline \multicolumn{3}{|l|}{ Cryptophaginae } \\
\hline Cryptophagus acutangulus Gyll & & East Azarbaijan, Fars, Northern Khorasan \\
\hline C. araxicola Reitter & & East Azarbaijan \\
\hline C. bruckii Reitter & & No locality cited \\
\hline C. cellaris (Scop.) & & East Azarbaijan, Hamadan, Tehran \\
\hline C. cellaroides Reitter & & Tehran \\
\hline C. dentatus (Hebst) & & Fars, Kuhgiloyeh \&Boyerahmad, Mazandaran, \\
\hline C. denticulatus Heer & & East Azarbaijan, Ilam \\
\hline C. dilutus Reitter & & No locality cited \\
\hline C. distinguendus Sturm & & East Azarbaijan, Mazandaran, \\
\hline C. erichsoni Reitter & & No locality cited \\
\hline C. hauseri Reitter & & No locality cited \\
\hline C. hexagonalis Tournier & & East Azarbaijan \\
\hline C. insulicola Roubal & & No locality cited \\
\hline C. intermedius Bruce & NI & Fars \\
\hline C. jakowlewi Reitter & & Fars, Mazandaran \\
\hline C. lapidicola Reitter & & No locality cited \\
\hline C. laterangulus Reitter & & No locality cited \\
\hline C. laticollis Lucas & & East Azarbaijan, Mazandaran, Tehran \\
\hline C. opacosericeus Reitter & NI & East Azarbaijan, \\
\hline C. pallidus Sturm & NI & Mazandaran \\
\hline C. punctipennis Bris. & & $\begin{array}{l}\text { Chaharmahal \& Bakhtiari, East Azarbaijan, Hamadan, Kerman, Kordestan, } \\
\text { Kuhgiloyeh \& Boyerahmad, Lorestan, Mazandaran, Tehran, }\end{array}$ \\
\hline C. quadrimaculatus Reitter & NI & Northern Khorasan \\
\hline C. scanicus (L.) & & Guilan, Mazandaran, Tehran \\
\hline C. scutellatus New. & & Mazandaran \\
\hline C. setulosus Sturm & NI & West Azarbaijan \\
\hline C. simplex Miller & & East Azarbaijan \\
\hline C. skalitzyi Reitter & & Isfahan, Mazandaran, Tehran \\
\hline Micrambe nigricollis (Reitter) & & Fars \\
\hline M. woodroffei Johnson & & Guilan \\
\hline \multicolumn{3}{|l|}{ Atomariinae } \\
\hline Atomaria (Atomaria) analis Erich & & No locality cited \\
\hline A. apicalis Erich. & & No locality cited \\
\hline A. atricapilla Steph. & & No locality cited \\
\hline A. fasciata Kol. & NI & Ardabil \\
\hline A. gravidula Erich. & & No locality cited \\
\hline A. lewisi Reitter & & No locality cited \\
\hline A. munda Erich. & NI & Razavi Khorasan \\
\hline A. peltata Kraatz & & No locality cited \\
\hline A. pusilla (Payk.) & & No locality cited \\
\hline A. rubricollis Bris. & & No locality cited \\
\hline Atomaria (Agathengis) nigrirostris Steph. & & No locality cited \\
\hline A. pulchra Erich. & & No locality cited \\
\hline A. vespertina Mäklin & & No locality cited \\
\hline Curelius exiguus (Erich.) & & No locality cited \\
\hline Ephistemus globulus (Payk.) & & No locality cited \\
\hline E. reitteri Casey & & No locality cited \\
\hline
\end{tabular}


HNHM); Fars province, Saadat-Shah, 1900 m, 20.IV.1999,

2 ex (leg. Nadai, HNHM).

General DistRIBUTION - Common in Europe, North Africa, Iran, central Asia, Far East, Nearctic Region (JOHNSON et al., 2007; OTERO, 2011; OTERO \& JOHNSON, 2013).

Host - The larva lives in the droppings of various xylophagous insects and on some species of Polyporus sp. (DONISTHORPE, 1939).

\section{Cryptophagus denticulatus Heer, 1841}

EXAMINED MATERIAL - East Azarbaijan province, $7 \mathrm{~km}$ NW Miyaneh, 12-13.X.2002, 1 ex (leg. P. Gyulai and A. Garani); Ilam province, Sarab, 26.VI.1974, 1 ex (leg. A. Senglet, MHNG).

General distribution - Europe, Morocco, Atlantic Islands (Madeira), Asian Turkey, Israel, Iran, Uzbekistan, Siberia, also Afrotropical, Australasian and Nearctic Regions (JOHNSON et al.; 2008; OTERO, 2011; 2013; OTERO \& JOHNSON, 2013).

\section{Cryptophagus dilutus Reitter, 1874}

DISTRIBUTION IN IRAN - Iran (no locality cited) (JOHNSON et al., 2007; LJUBARSKY \& PERKOVSKY, 2011).

GENERAL DISTRIBUTION - Europe, North Africa, Asia and Nearctic Region (JoHnson et al., 2007; LJUBARSKY \& PERKOVSKY, 2011; Otero, 2013).

\section{Cryptophagus distinguendus Sturm, 1845}

Distribution IN IRAN - East Azarbaijan (MOdARRes AWAL, 1997).

NEW DATA - Mazandaran province, Gol-e-Loveh, 1200 m, 21.VIII.1975, 3 ex (leg. A. Senglet, MHNG).

General Distribution - Asia, Europe, North Africa, Nearctic and Afrotropical Regions (JOHNSON et al., 2007; OTERO, 2011; 2013).

Host - Fungus and on dried fruits (in damp granaries) (ModArRes AwAL, 1997).

\section{Cryptophagus erichsoni Reitter, 1888}

DISTRIBUTION IN IRAN - Iran (no locality cited) (JOHNSON et al., 2007).

General Distribution - Romania, Russia, Armenia, Azerbaijan, (JOHNSON et al., 2007; OTERO, 2013).

\section{Cryptophagus hauseri Reitter, 1890}

DISTRIBUTION IN IRAN - Iran (no locality cited) (JOHNSON et al., 2007).

General Distribution - Central and South European Territory (Kavkaz), Armenia, Iran, Central Asia, Xinjiang (Sinkiang), Ukraine (JoHnson et al., 2007).

\section{Cryptophagus hexagonalis Tournier, 1872}

Distribution In Iran - East Azarbaijan (Modarres AWAL, 1997).

General distribution - Finland, Belarus, Russia, Turkey, Siberia, Mongolia, Kazakhstan, Turkmenistan (JOHNSON et al., 2007; OTERO, 2013).

Host - Fungus and on dried fruits (in damp granaries) (Modarres Awal, 1997).

\section{Cryptophagus insulicola Roubal, 1919}

DISTRIBUTION IN IRAN - Iran (no locality cited) (JOHNSON et al., 2007; OTERO \& JOHNSON, 2013).

GENERAL Distribution - Species very rare in Europe. Great Britain, Germany, Greece, Switzerland, Afghanistan, Iran, Tajikistan, Turkmenistan, Uzbekistan (JOHNSON et al., 2007; OtERo, 2013; OtERo \& JOHNSON, 2013).
Cryptophagus intermedius Bruce, 1934

EXAMINED MATERIAL - Prov. Fars, Sadat-Shah; 53 $12^{\prime}$ 38" E 30 5' 21" N., 1900 m; 20.IV1999, 1 ex (leg. Meszaros-Zombori, HNHM).

GENERAL DISTRIBUTION - Rare but widely distributed in Europe. Austria, Denmark, Estonia, France, Great Britain, Germany, Georgia, Italy, Norway, Poland, Romania, Slovakia, South of Russia (Kavkaz), Spain, Sweden, Switzerland, Azerbaijan, Armenia (JoHNSON et al., 2007; OTERO, 2011; OTERO \& JOHNSON, 2013).

COMMENTS - New record for Iran.

\section{Cryptophagus jakowlewi Reitter, 1888}

EXAMINED MATERIAL - Mazandaran province, Tang-eRah, 16.VII.1975, 3 ex (leg. A. Senglet, MHNG); Fars province, $3 \mathrm{~km}$ W Sangas, $52^{\circ} 1$ ' 8 "' E $30^{\circ} 11^{\prime} 4$ " N, 11.IV.1999, 1 ex; Fars province, Saadat-Shahr, 1900 m, 20.IV.1999 (leg. Fabian-Nadai, HNHM).

GENERAL DISTRIBUTION - Europe, North Africa, Caucasus, Turkey, Central Asia (Kirghizia, Iran), East Siberia (LJUBARSKY, 2002; JOHNSON et al., 2007; OTERO, 2011; 2013)

\section{Cryptophagus lapidicola Reitter, 1879}

DISTRIBUTION IN IRAN - Iran (no locality cited) (JOHNSON et al., 2007).

General Distribution - Armenia, Azerbaijan, Georgia, South Russia, Turkey (Johnson et al., 2007; OTERo, 2011; 2013).

\section{Cryptophagus laterangulus Reitter, 1891}

DISTRIBUTION IN IRAN - Iran (no locality cited) (JOHNSON et al., 2007; LJUBARSKY \& PerKovsky, 2011).

General distribution - South Russia, Iran, Iraq, Kazakhstan, Turkmenistan (JoHnson et al., 2007; LJUBARSKY \& PERKovsky, 2011; OTERO, 1996; 2013).

Cryptophagus laticollis Lucas, 1846

DisTRIBUTION IN IRAN - East Azarbaijan (MODARRES AWAL, 1997)

NEW DATA - Mazandaran province, Chortheh, $1600 \mathrm{~m}$, (VII.1973, 7 ex; Tehran province, Pol-e-Zanguleh, 230 m, 12.VII, 1973, 1 ex (leg. A. Senglet, MHNG).

General Distribution - Europe, all North Africa, Atlantic Islands, Afganistan, India, Russia, Israel, Iraq, Jordania, Kyrgystan, China, Syria, Turkmenistan, Turkey, Uzbekistan, India, Afrotropical, Nearctic, Australian and Oriental Regions (JOHNSON et al., 2007; LJUBARSKY, 1997; OTERO, 1997; 2011; 2013; OTERO et al., 2001;).

Host - Fungus and on dried fruits (in damp granaries) (ModARres AwAL, 1997).

Cryptophagus opacosericeus Reitter, 1896

EXAMINED MATERIAL - East Azarbaijan province, $7 \mathrm{~km}$ NW Miyaneh, 12-13.X.2002, 1 ex (leg. Gyulai-Garai, HNHM).

General Distribution - Albania, South European Territory Russia, Azerbaijan, Kazakhstan, Kyrgyzstan, Tadzhistan (JOHNSON et al., 2007; OTERO, 2013).

COMMENTS - New record for Iran.

Cryptophagus pallidus Sturm, 1845

EXAMINED MATERIAL - Mazandaran province, Tang-eRah, 16.VII.1975, 1 ex; Mazandaran province, Gol-eLoweh, 700 m, 21.VII.1975, 3 ex; Mazandaran province, Shahps Sand, 28. VII.1974, 1 ex (leg. A. Senglet, MHNG). 
General Distribution - Europe, North Africa, Turkey, Israel, Lebanon, Iran (JOHNSON et al., 2007; OTERO, 2011; 2013).

COMMENTS - New record for Iran.

Cryptophagus punctipennis C. N. F. Brisout de Barneville, 1863

EXAMINED MATERIAL - East Azarbaijan province, Bonab, 4.VI.1975, 10 ex; East Azarbaijan province, Miyaneh, 7.VI.1975, 1 ex; Chaharmahal \& Bakhtiari province, barrage Kührang, 18.VI.1974, 5 ex; Chaharmahal \& Bakhtiari province, route Kührag, 17.VI.1974, 6ex; Hamadan province, 29.VII.1975, 1 ex; Kerman province, 4.VIII.1973, 1 ex; Northern Khorasan province, Esfarayen, 1200 m, 20.VII.1975, 2 ex; Kuhgiloyeh \& Boyerahmad province, route Yasuj, 220 m, 27.V.1974, 1 ex; Kordestan province, Sanandaj, 21.Vi.1975, 2 ex; Lorestan province, Azna, 23.VI.1974, 3 ex; Lorestan province, Ma'amulan, 24.VI.1974, 1 ex; Lorestan province, Borudjerd, 10.IX.1975, 2 ex; Mazandaran province, Tang-e-Rah, 16.VII.19075, 1 ex; Mazandaran province, Gol-e-Loweh, 700 m, 21.VIII.1975, 1 ex; Tehran province, Disine, 3500 m, 13.VIII.1973, 1 ex; Tehran province, Kendvan, 12.VII.1973, 1 ex; Tehran province, Pol-e-Djadjirud, 16.VII.1973, 1 ex; Tehran province, Polur, $2300 \mathrm{~m}$, 17.VII.1973, 1 ex (leg. A. Senglet, MHNG).

GENERAL DISTRIBUTION - It is possibly a cosmopolitan species, in greater or lesser degree, Europe, North Africa, Atlantic Islands, Asia from Turkey and Iran to Japan, Australian and Nearctic Regions (JoHNSON et al., 2007; OTERo, 2011; 2013; WOODROFFE \& COOMBS, 1961).

Cryptophagus quadrimaculatus Reitter, 1877

EXAMINED MATERIAL - Northern Khorasan province, 70 km NE Qucan, 2200 m, 10.V.2001, 1 ex; 12.V.2001, 8 ex; 13.V.2001, 1 ex (leg. Bendek \& Csorba, MHNG).

General distribution - Finland, Central and South European Territory Russia, Armenia, Azerbaijan, Georgia, Siberia, Iraq, Kyrgyzstan, Mongolia, Syria, Tadzhikistan, Turkmenistan, Uzbekistan, Nearctic Region (JoHnsON et al., 2007; LJUBARSKY, 2002; OTERO, 2013).

COMMENTS - New record for Iran.

Cryptophagus scanicus (Linnaeus, 1758)

DISTRIBUTION IN IRAN - Iran (no locality cited) (MAJKA \& LANGOR, 2010).

NEW DATA - Mazandaran province, Tang-e-Rah, 16.VII.1975, 7 ex; Mazandaran province, Gol-e-Loweh, 21.VIII.1975, 700 m, 2 ex; Mazandaran province, Kiyasar, 1500 m, 11.VII.1975, 6 ex; Guilan province, Siahkal, 6.VII.1973, 2 ex; Tehran province, Kendran, 2700 m, 12.VII.1973, 1 ex (leg. A. Senglet, MHNG).

GENERAL Distribution - Specie Holartic: Europe, North Africa in Tunisia and Madeira, Asia in Turkey, Iran and Siberia, Afrotropical and Nearctic Regions (JoHNSON et al., 2007; LJUBARSKY, 2002; OTERO, 2011; 2013).

Cryptophagus scutellatus Newman, 1834

EXAMINED MATERIAL - Mazandaran province, route Tchorteh, 1300 m, 5.VIII.1974, 1 ex (leg. A. Senglet, MHNG).

General Distribution - Europe, North Africa (Morocco), Asian Turkey, Iran, Nearctic Region (JOHNSON et al., 2007; LJUBARSKY, 2002).

\section{Cryptophagus setulosus Sturm, 1845}

EXAMINED MATERIAL - West Azarbaijan province,
Ourmieh, Zeynaloo, 37³7'57'N 450'22”'E, $1612 \mathrm{~m}$,

13.IX.2007, 2 ex.

General Distribution - Europe, North Africa, Turkey, Kyrgyzstan. Kazakhstan, Afghanistan, East Siberia (JOHNSON et al., 2007; OTERO, 2013).

COMMENTS - New record for Iran

Cryptophagus simplex Miller, 1858

Distribution IN IRAN - East Azarbaijan (ModArRes AWAL, 1997).

General Distribution - Austria, Czech Republic, Great Britain, Germany, Hungary, The Netherlands, South Russia, Azerbaijan, Armenia, Kyrgyzstan, Uzbekistan (JOHNSON et al. 2007).

Host - Fungus and on dried fruits (in damp granaries) (MODARRES Awal, 1997).

\section{Cryptophagus skalitzkyi Reitter, 1875}

EXAMINED MATERIAL - Isfahan province, Eskandari, 8.VII.1973, 1 ex; Mazandaran province, Baladeh, 2200 m, 12.VII.1974; Mazandaran province, Tang-e-Rah, 16.VII.1975, 2 ex; Tehran province, Disine, $3500 \mathrm{~m}$, 13.VII.1973, 1 ex (leg. A. Senglet, MHNG).

General distribution - Germany, Croatia, Spain, France, Great Britain, Italy, Czech Republic, Armenia, Azerbaijan, Georgia Turkey, Iran, Turkmenistan, Uzbekistan, Tajikistan, Kyrgyzstan, Kazakhstan, Afghanistan, Pakistan, India, Eastern Siberia (JoHNSON et al., 2007; LJUBARSKY \& PERKOVSKY, 2011; Otero, 2013).

Micrambe (Micrambe) nigricollis (Reitter, 1876)

EXAMINED MATERIAL - Fars province, Izad Khast, 16.VIII.1973, 1 ex (leg. A. Senglet. MHNG).

General Distribution - Albania, Russia, Azerbaijan, Armenia, Georgia, Iran, Kyrgyzstan. Kazakhstan, Tadzhikistan, Mongolia, East Siberia (JOHNSON et al. 2007; OTERO, 2013).

Micrambe (Micrambe) woodroffei Johnson, 2007

Examined material - Guilan province, Pareh-Sar, 2.VIII. 1973, 1 ex (leg. A. Senglet, MHNG).

General Distribution - Europe, North Africa, Cyprus, Turkey, Iran, Lebanon, Israel (JoHnSON et al., 2007; OTERO, 2013).

Subfamily Atomariinae LeConte, 1861

Atomaria (Atomaria) analis Erichson, 1846

DISTRIBUTION IN IRAN - Iran (no locality cited) (JOHNSON et al., 2007).

General Distribution - All Europe, Turkey, Iran (JOHNSON et al., 2007; LJUBARSKY, 2014).

Atomaria (Atomaria) apicalis Erichson, 1846

DISTRIBUTION IRAN - Iran (no locality cited) (JOHNSON, 1993; JOHNSON et al., 2007).

GENERAL DISTRIBUTION - It is widely distributed throughout Europe and occurs in the Atlantic Islands, Turkey, Israel, Iran, Siberia, Mongolia, Central Asia, North Africa and North America (Johnson, 1993; JoHnson et al., 2007).

Atomaria (Atomaria) atricapilla Stephens, 1830

DISTRIBUTION IN IRAN - Iran (no locality cited) (JOHNSON et al., 2007).

General distribution - Europe, Morocco, Tunisia, 
Cyprus, Turkey, Iran, Jordan, Syria, Israel (JoHnson et al., 2007).

Atomaria (Atomaria) fasciata Kolenati, 1846

EXAMINED MATERIAL - Ardabil province, Germi, 3900'N $47^{\circ} 57^{\prime} \mathrm{E}$, June 2010, 1 ex.

GeNERAL DisTRIBUTION - Central and south Europe, North Africa (with Canary Islands), Central Asia, China (JoHNSON et al., 2007).

COMMENTS - New record for Iran.

Atomaria (Atomaria) gravidula Erichson, 1846

DISTRIBUTION IN IRAN - Iran (no locality cited) (JOHNSON et al., 2007).

GeNERAL DisTRIBUTION - Europe (not Iberian Peninsula and the north), Turkey, Iran (JoHNSON et al., 2007).

Atomaria (Atomaria) lewisi Reitter, 1877

DISTRIBUTION IN IRAN - Iran (no locality cited) (JOHNSON et al., 2007).

GENERAL DISTRIBUTION - Cosmopolitan species; Europe (not Iberian Peninsula), Asia (JoHnson et al., 2007; LJUBARSKY, 2014).

Atomaria (Atomaria) munda Erichson, 1846

EXAMINED MATERIAL - Razavi Khorasan province, Taybad, $34^{\circ} 48^{\prime} \mathrm{N} 60^{\circ} 40^{\prime} \mathrm{E}, 800 \mathrm{~m}$, April 2011, 2 ex.

GENERAL DISTRIBUTION - It is widely distributed all over Europe apart from the extreme north, the Atlantic Islands and North Africa and is also known from Turkey, Israel, Afghanistan, Kazakhstan (JoHnson, 1993; JoHnsON et al., 2007).

COMMENTS - New record for Iran.

Atomaria (Atomaria) peltata Kraatz, 1853

DISTRIBUTION IN IRAN - Iran (no locality cited) (JOHNSON, 1993; JOHNSON et al., 2007).

GENERAL DISTRIBUTION - It is widely distributed all over Europe, except the south-west, but is commonest in the east. It occurs in Iran, Kazakhstan, Uzbekistan, Afghanistan, Siberia, Mongolia, Xinjiang (Sinkiang) (JoHnSON, 1993; JOHNSON et al., 2007).

Atomaria (Atomaria) pusilla (Paykull, 1798)

DISTRIBUTION IN IRAN - Iran (no locality cited) (JOHNSON, 1993; JOHNSON et al., 2007).

General Distribution - It is widely distributed throughout most part of Europe, North Africa, the Atlantic Islands and also occurs in Turkey, Iran, Afghanistan, Kazakhstan, Mongolia, Far East and North America (JoHnson, 1993; JoHnson et al., 2007).

Atomaria (Atomaria) rubricollis Brisout de Barneville, 1863

DISTRIBUTION IN IRAN - Iran (no locality cited) (JOHNSON, 1993; JOHNSON et al., 2007).

GENERAL DISTRIBUTION - There are records from scattered localities over much of Europe. It is also known from Turkey, Cyprus, Syria, Israel, Jordan, Iran and Central Asia (JoHNSON, 1993; JOHNSON et al., 2007).

\section{Atomaria (Agathengis) nigrirostris Stephens, 1830}

DISTRIBUTION IN IRAN - Iran (no locality cited) (JOHNSON, 1993; JOHNSON et al., 2007).

General Distribution - It is widely distributed throughout Europe, Algeria and also occurs in Siberia, Turkey, Iran, Central Asia, Mongolia and North America (JoHNSON, 1993; JoHnSON et al., 2007).
Atomaria (Agathengis) pulchra Erichson, 1846

DisTRIBUTION IN IRAN. Iran (no locality cited) (JOHNSON, 1993).

General distribution - Europe (not in Iberian Peninsula), Caucasus, Turkmenistan, Afghanistan (JoHNSON et al., 2007; LJUBARSKY, 2014).

\section{Atomaria (Agathengis) vespertina Mäklin, 1853}

DISTRIBUTION IN IRAN - Iran (no locality cited) (JOHNSON et al., 2007).

GENERAL DISTRIBUTION - Europe, Canary Islands, Turkey, Iran, Siberia, Japan, Mongolia, Nepal, India (JoHnson et al., 2007; LJUBARSKY, 2014).

Curelius exiguus (Erichson, 1846)

DISTRIBUTION IN IRAN - Iran (no locality cited) (JOHNSON et al., 2007).

General distribution - Europe, North Africa, Cyprus, Turkey, Israel, Jordan, Iran, Iraq, Tajikistan (JoHNSON et al., 2007).

\section{Ephistemus globulus (Paykull, 1798)}

DISTRIBUTION IN IRAN - Iran (no locality cited) (JOHNSON, 1993; JOHNSON et al., 2007).

General Distribution - This species is widely distributed throughout Europe but it is more scattered in the South. It also occurs in North Africa and the Atlantic islands, as well as Iran, Kazakhstan, Northern China, Mongolia, Far East, New Zealand and North America (JOHNSON, 1993; JoHNSON et al., 2007).

Ephistemus reitteri Casey, 1900

DISTRIBUTION IN IRAN - Iran (no locality cited) (JOHNSON et al., 2007; OTERO et al., 2001).

GENERAL DISTRIBUTION - Europe except west and northeast, Cyprus, Israel, Turkey, Iran (JoHnson et al., 2007; OTERO et al., 2001).

\section{DISCUSSION}

\section{FAUNISTIC COMPOSITION}

A total of 46 species from 5 genera and 2 subfamilies, Cryptophaginae (29 species from 2 genera) Atomariinae (17 species from 3 genera) and are listed as the fauna of Iranian Cryptophagidae (Table 1). Seven species are newly recorded for the fauna of Iran: Atomaria (Atomaria) fasciata Kolenati, 1846, Atomaria (Atomaria) munda Erichson, 1846 (Subfamily Atomariinae), Cryptophagus intermedius Bruce, 1934, Cryptophagus opacosericeus Reitter, 1896, Cryptophagus pallidus Sturm, 1845, Cryptophagus quadrimaculatus Reitter, 1877, Cryptophagus setulosus Sturm, 1845 (Subfamily Cryptophaginae). The list of species is given below alphabetically with distribution data.

Recoding of 14 new records indicates that the fauna of Iranian Cryptophagidae is diverse. Among the 5 recorded genera, Cryptophagus with 29 species is more diverse than the other genera, and consequently is Atomaria with 3 genera. Since Iran is a large country with various geographical regions and climates, conducting the faunistic surveys on Cryptophagidae is necessary in different regions of Iran for finding new data. Additionally, some species of Cryptophagidae have powerful parasitoids which have an efficient role in decreasing their population density. Larvae of beetles of the families Curculionidae, Anobiidae, and Cryptophagidae are the most frequently recorded hosts of Blacus species (Hymenoptera: Braconidae: Blacinae) 
(ACHTERBERG VAN, 1988; YU et al., 2012). Investigation on this host-parasitoid relationship will result to valuable findings.

\section{ACKNOWLEDGEMETS}

The redearch was supported by Islamic Azad University (Yadegar - e-Imam Khomeini (RAH) Branch).

\section{REFERENCES}

ACHTERBERG C. VAN, 1988 - Revision of the subfamily Blacinae Foerster (Hymenoptera, Braconidae). - Zool. Verh. (Leiden), 249: 1-324.

Bartolozzi L., Ghahari H., Sprecher-Uebersax E., ZILIOLI M., 2014 - A checklist of stag beetles (Coleoptera: Scarabaeoidea: Lucanidae) from Iran. - Zootaxa, 3887 (3): 422-436.

Bunalski M., Samin N., Ghahari H., Hawkeswood T.J., 2014 - Contributions to the knowledge the scarab beetles of Golestan province, Northern Iran with checklist of Iranian Scarabaeoidea (Coleoptera). - Polish J. Entomol., 83: 141-170.

DONISTHORPE H., 1939 - A preliminary list of the Coleoptera of Windsor Forest. Nathaniel Lloyd \& Co. London 126 pp.

Ghahari H., Volkovitsh M., Bellamy C.L., 2015 - An annotated catalogue of the Buprestidae of Iran (Coleoptera: Buprestoidea). - Zootaxa, 3984 (1), 1-141.

GHAHARI H., NOVAK V., 2015 - A checklist of comb-clawed beetles (Coleoptera: Tenebrionidae: Alleculinae) from Iran. - Zootaxa, 4027 (1): 101-116.

Johnson C., 1993 - Provisional atlas of the Cryptophagidae-Atomariinae (Coleoptera) of Britain and Ireland. Biological Records Centre, Huntingdon, UK. 91 pp.

Johnson C., Otero J. C., Leschen R.A.B., 2007 - New Acts \& Comments [Esser, J.], pp. 513-531, Catalogue Cryptophagidae. In: Löbl, I. \& Smetana, A. (Eds.), Catalogue of Palaearctic Coleoptera, Vol. 4: 65-70. Apollo Books, Stenstrup, Kopenhagen, Denmark, 935 pp.

Johnson C., Otero J. C., Leschen R.A.B., 2008 - Errata for volume 3, Cryptophagidae, pp. 26-27. In: Löbl, I. \& Smetana, A. (eds.), Catalogue of Palaearctic Coleoptera, Vol. 5. Apollo Books, Stenstrup, Kopenhagen, Denmark. $670 \mathrm{pp}$.

Lason A., Ghahari H., 2013 - A checklist of the Kateretidae and Nitidulidae of Iran (Coleoptera: Cucujoidea). - Zootaxa, 3746 (1): 101-122.

Legalov A. A., Ghahari H., Arzanov Yu G., 2010 Annotated catalogue of curculionid-beetles (Coleoptera: Anthribidae, Rhynchitidae, Attelabidae, Brentidae, Brachyceridae, Dryophthoridae and Curculionidae) of Iran. - Amurian Zool. J., II (3): 191-244.

LESCHEN R.A.B., 1999 - Origins of symbiosis: phylogenetic patterns of social insect inquilinism in Cryptophagidae (Coleoptera: Cucujoidea). University of Kansas Museum of Natural History. Special Publication, 24: 85-101.

LJUBARSKY G. YU, 1992 - Caucasian silken fungus beetles from the genus Cryptophagus. - Zool. Zh., 71 (10): 68-82 [in Russian].
LJUBARSKY G. Yu, 1997 - Cryptophagidae and Languriidae from India (Coleoptera, Clavicornia). Entomofauna, 18 (5): 49-60.

LJUBARSKY G. YU, 2000 - New and rare species of the family Cryptophagidae from northern Palaearctic (former USSR) (Coleoptera: Clavicornia). - Russ. Entomol. J., 9 (4): 321-328.

LJUBARSKY G. Yu, 2002 - [Cryptophaginae (Coleoptera: Cucujoidea: Cryptophagidae): Diagnostics, Arealogy,Ecology]. Moscow University Publisher. Moscow. $421 \mathrm{p}$.

LJUBARSKY G. Yu, 2014 - Cryptophagidae (Coleoptera: Clavicornia) from China and adjacent regions. Russ. Entomol. J., 23 (1): 19-40

Ljubarsky G.Yu, Perkovsky E.E., 2011 - Third contribution on Rovno amber silken fungus beetles: a new Eocene species of Cryptophagus (Coleoptera, Clavicornia, Cryptophagidae). In: Shcherbakov, D.E., Engel, M.S. \& Sharkey, M.J. (eds), Advances in the systematics of fossil and modern insects: Honouring Alexandr Rasnitsyn. ZooKeys, 130: 255-261.

MAJKa C. G., LANGOR D., 2010 - Contributions towards an understanding of the Cryptophaginae (Coleoptera, Cryptophagidae) of Atlantic Canada. - ZooKeys, 35: 13-35.

Modarres Awal M., 1997 - Family Cryptophagidae, p. 161. In: Modarres Awal, M. (ed.), List of agricultural pests and their natural enemies in Iran. Ferdowsi University Press, Mashhad, 497 pp.

Otero J.C., 1996 - Sobre la identidad de tres especies del género Cryptophagus Herbst, 1792 (Coleoptera: Cryptophagidae). $1^{\mathrm{a}}$ nota. Elytron, 10: 5-10.

OTERO J.C., 1997 - Three new species and distributional records of Micrambe CG. Thomson, 1863 and Cryptophagus Herbst, 1792 (Coleoptera: Cryptophagidae) from Israel and Turkey. - Rev. Suisse Zool., 104 (1): 207-216.

Otero J.C., 2011 - Coleoptera, Monotomidae, Cryptophagidae. En: Fauna Iberica, vol. 35. Ramos M.A. et al. Museo Nacional de Ciencias Naturales. CSIC. Madrid, $365 \mathrm{pp}$.

OTERo J.C., 2013 - Cryptophaginae (Coleoptera) de la Región Paleártica occidental. - Coleop. Monographs, 4: 1-296.

Otero J.C., Johnson C., Mifsud D., 2001 - Cryptophagids from the Maltese Islands with description of a new species of Micrambe Thomson (Coleoptera: Cryptophagidae). Koleop. Rund., 71: 163-170.

OTERO J.C., JOHNSON C., 2013 - Species of Cryptophagus Herbst, 1792, belonging to the "dentatus group" (Coleopera: Cryptophagidae) from the Western Palearctic region. -Entomol. Fenn., 24: 81-93.

Woodroffe G.E., Coombs C.W., 1961 - Revision of the North American Cryptophagus Herbst (Coleoptera: Cryptophagidae). - Misc. Publ. Entomol. Soc. Am., 2 (2): 179-211.

Yu D.S., Achterberg C. Van, Horstmann K., 2012 World of Ichneumonoidea 2011. Biological and taxonomical information), Taxapad Interactive Catalogue, Ottawa. Available from: ww.taxapad.com

Zehzad B., Kiabi BH., Madjnoonian H., 2002 - The natural areas and landscape of Iran: an overview. - Zool. Midd. East, 26: 7-10. 
52 - Blank Page 